\title{
Cylindrical crystalline-core micelles: pushing the limits of solution self-assembly
}

\author{
Joachim Schmelz, ${ }^{a}$ Felix H. Schacher ${ }^{\star b}$ and Holger Schmalz ${ }^{* a}$ \\ Within the last few years, crystallisation-induced self-assembly of block copolymers (BCs) in solution has \\ become more and more attractive for the production of well-defined cylindrical crystalline-core micelles \\ (cCCMs). The "livingness" of this process has been shown for a number of crystallisable blocks and \\ allows fine tuning of the length as well as the length distribution of such structures. This \\ unprecedented control is hardly achievable in the self-assembly of purely amorphous BCs. Furthermore, \\ in an analogy to living/controlled polymerisation methods, the epitaxial growth of different BCs onto \\ preformed cCCMs allows for the preparation of complex micellar architectures, e.g., cylindrical block co- \\ micelles. This highlight tries to provide an overview over recent developments in crystallisation-induced \\ self-assembly of BCs with a particular focus on one-dimensional (1D) micellar nanostructures.
}

Received 1st October 2012

Accepted 6th December 2012

DOI: $10.1039 / \mathrm{c} 2 \mathrm{sm} 27259 \mathrm{~h}$

www.rsc.org/softmatter

nanometer scale. Over the last few decades intensive studies revealed a multitude of morphologies that are accessible via segregation of these polymer blocks in the bulk phase or in solution. ${ }^{1-6}$ Self-assembled BC micelles are used in numerous applications such as emulsion polymerisation, ${ }^{7}$ drug delivery, ${ }^{8}$ compatibilisation of polymer blends, ${ }^{9-11}$ membrane production, ${ }^{12}$ or as nanoreactors in synthesis/catalysis. ${ }^{13,14}$ In the case of amorphous (coil-coil) BCs in solution, quite profound knowledge on how to predict structure formation already exists. ${ }^{15-17}$ Still, most structures exhibit a spherical morphology,

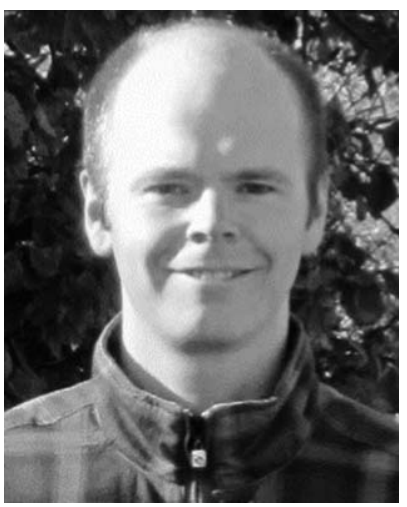

Joachim Schmelz was born in 1982 in Lauterbach, Germany. Including internships at the LCPO (ENSCP de Bordeaux, France) and Bayer Technology Services (Leverkusen, Germany), he graduated in polymer chemistry at the University of Bayreuth in 2008. During his $P h D$ with Prof. Axel H. E. Müller and Dr Holger Schmalz he focused on the crystallisation-driven self-assembly of polyethylene containing block copolymers to one-dimensional patchy micelles. Recently, he joined Funktronic GmbH (Wartenberg, Germany) as a software developer for radio control panels in polymer housings.

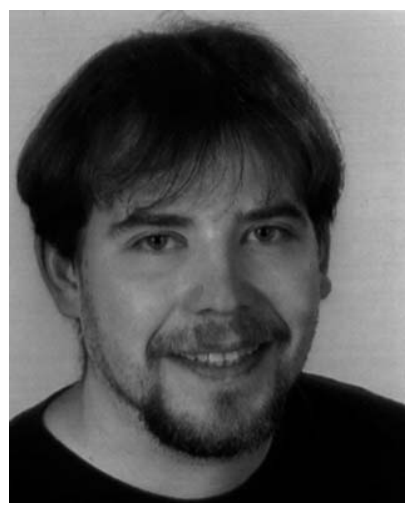

Felix H. Schacher was born in Lichtenfels, Germany, in 1980. After finishing his graduate studies in chemistry at the University of Bayreuth, Germany in 2006, he completed his doctoral work under the supervision of Prof. Axel H. E. Müller in 2009. His thesis focused on living anionic polymerisation techniques and functional nanostructured systems based on block co- and terpolymers in the bulk and in solution. Afterwards, he joined the group of Prof. Ian Manners as a DAAD postdoctoral fellow, working on the synthesis and self-assembly of organometallic block copolymers. Since 2010, he has been a junior professor at the Friedrich-SchillerUniversity Jena, Germany. His research interests are polymer synthesis, self-assembly of polymer-based materials, and different characterisation techniques related to block copolymer nanostructures. 
whereas cylindrical micelles are typically only stable in the socalled "crew-cut" regime, where the core-forming block is significantly longer than the corona forming block, or in specific solvent mixtures..$^{\mathbf{5 1 8 , 1 9}}$ In other approaches, cylindrical superstructures could be produced from pre-formed micellar building blocks via stepwise solvent exchange or by using suitable additives. ${ }^{\mathbf{2 0 , 2 1}}$ The crosslinking of polymer blocks in cylindrical bulk phases followed by sonication-assisted re-dispersion in non-selective solvents is another method for the preparation of $1 \mathrm{D}$ micelles. ${ }^{22-24}$

The solution behaviour of BCs with crystallisable blocks on the other hand has not been investigated to a similar extent, partly due to the higher complexity of processes occurring during structure formation. In a previous theoretical work, Vilgis and Halperin used a chain-folding model to describe the self-assembly of BCs into crystalline-core micelles (CCMs). ${ }^{25}$ The resulting morphology, i.e., the morphology showing the lowest total free energy, is mainly determined by three competing factors. ${ }^{25,26}$ A low amount of chain folds is advantageous in view of the crystallisation enthalpy. However, at the same time this leads to a higher grafting density and, hence, stretching of the soluble corona chains, which is entropically unfavourable. Additionally, a minimisation of the high energy crystal surface results in a lower free energy. Even though spherical, cylindrical, and platelet-like CCMs were predicted, early investigations of the self-assembly of crystalline-coil BCs revealed almost exclusively platelet-like structures. ${ }^{26-30}$ Herein, we focus on recent developments regarding the solution-based formation of $1 \mathrm{D}$ nanostructures with a crystalline core-forming block. We put particular emphasis on different strategies that were used to control length, thickness, or composition (core and/or corona) of such structures. An excellent and more comprehensive review on semi-crystalline block copolymer nanostructures in the bulk and solution has been recently provided by $\mathrm{Xu}$ and He. ${ }^{31}$

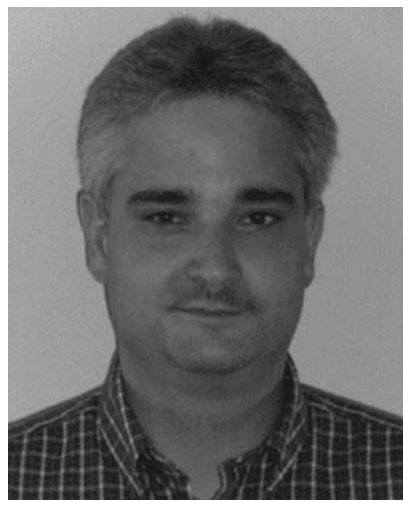

Holger Schmalz studied chemistry at the University of Bayreuth, Germany, and completed his PhD with Prof. Volker Abetz in 2002 on thermoplastic elastomers based on semicrystalline block copolymers. He then joined Ticona GmbH in Frankfurt, Germany, working on cationic polymerisation of trioxane and impact-modified polyoxymethylene. Since 2004, he has been a staff scientist (Akademischer Oberrat) in the department of Macromolecular Chemistry II at the University of Bayreuth. His research interests include block copolymer synthesis via living/controlled polymerisation methods, block copolymer self-assembly and stimuli-responsive organo- and hydrogels.

\section{Cylindrical crystalline-core micelles (cCCMs)}

In the late 90s Winnik, Manners et al. produced cCCMs from a poly(ferrocenyl dimethylsilane)-block-poly(dimethyl siloxane) (PFDMS- $b$-PDMS) diblock copolymer via dissolution of a hexagonally packed cylindrical bulk structure in $n$-hexane. ${ }^{32}$ Further studies revealed that the formation of cylinders for such systems occurred not only by direct dissolution of the bulk structure but also in various $n$-alkanes and by dialysis from the common solvent THF. ${ }^{33}$ The crystallisation of the PFDMS segment was identified as the main driving force for the formation of anisotropic micellar structures, even if compositions were used, where the formation of star-like micelles would be expected. If PFDMS was replaced by non-crystalline poly(ferrocenyl methylphenylsilane) or poly(ferrocenyl methylethylsilane), or if self-assembly was carried out above the melting point of PFDMS, spherical micelles were formed,
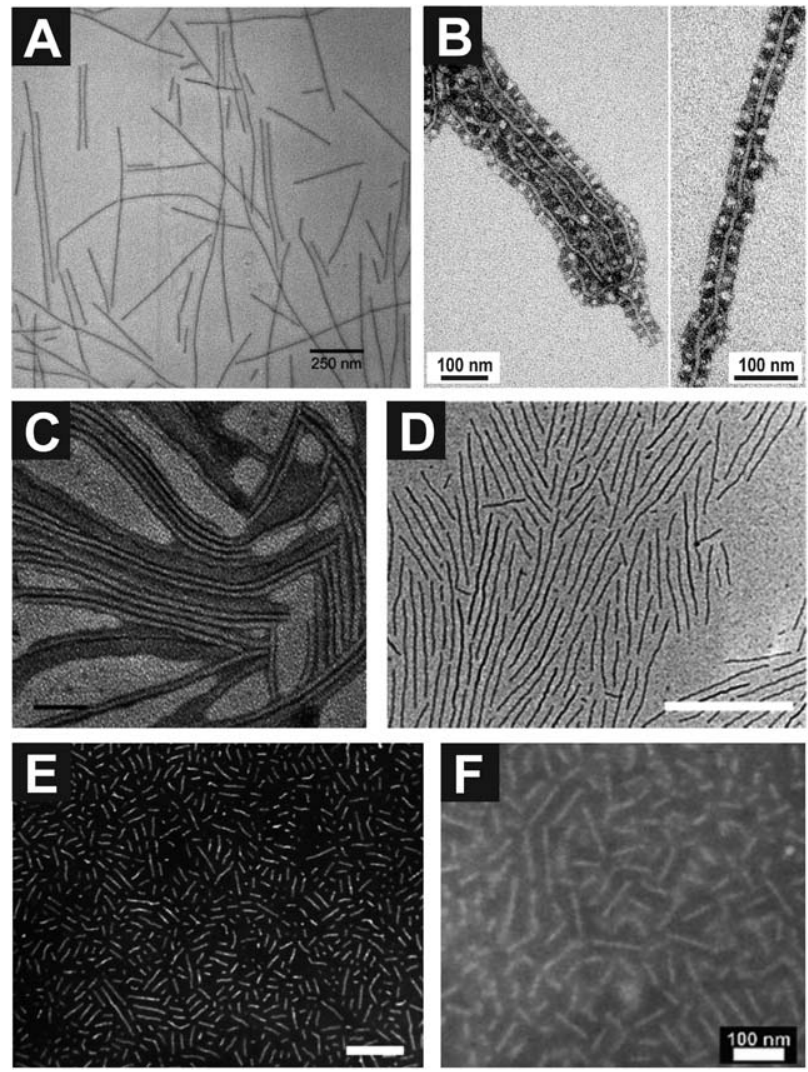

Fig. 1 Cylindrical micelles from Pl-b-PFS diblock copolymers (A, reprinted with permission from ref. 34; Copyright 2002 American Chemical Society); coronacompartmentalised cylindrical micelles from PS- $b$-PE- $b$-PMMA triblock terpolymers ( $B$, reprinted with permission from ref. 39; Copyright 2011 American Chemical Society); micelles from PCL-b-PEO block copolymers ( $C$, reprinted with permission from ref. 40; Copyright 2010 John Wiley and Sons); cylinders with a conjugated P3HT core (D, reprinted with permission from ref. 41; Copyright 2011 American Chemical Society); cylindrical micelles obtained from PI-b-PLA block copolymers ( $E$, adapted from ref. 42 with permission; Copyright 2011 the Royal Society of Chemistry); structures generated via solution self-assembly of PB- $b$-PEO block copolymers ( $F$, adapted from ref. 43 with permission; Copyright 2012 the Royal Society of Chemistry). 
further supporting this assumption. Cylindrical CCMs could also be produced when PDMS was substituted by different corona blocks such as polyisoprene (PI, Fig. 1A), ${ }^{34}$ poly(methyl methacrylate) (PMMA), ${ }^{35}$ poly(2-vinylpyridine) (P2VP), ${ }^{36}$ poly(ethylene oxide) (PEO), ${ }^{37}$ or $\operatorname{poly}(N, N$-dimethylaminoethyl methacrylate) (PDMAEMA), ${ }^{38}$ pointing out the extraordinary robustness of this $1 \mathrm{D}$ structure formation. Due to the hydrophilic outer blocks the latter cCCMs could even be transferred to water whilst retaining their morphology.

In later years, BCs with several crystallisable blocks were found to form 1D CCMs. ${ }^{44}$ Besides the PFDMS analogues poly(ferrocenyl dimethylgermane) (PFDMG) ${ }^{45}$ and poly(ferrocenyl diethylsilane) (PFDES), ${ }^{\mathbf{4}}$ cylindrical or worm-like CCMs were also produced via the self-assembly of poly( $\varepsilon$-caprolactone) (PCL, Fig. 1C), ${ }^{\mathbf{4 0 , 4 7}}$ stereoregular polylactides (srPLA, Fig. 1E), ${ }^{42,48,49}$ poly(ethylene oxide) (PEO, Fig. 1F) ${ }^{\mathbf{4 3 , 5 0}}$ and polyacrylonitrile $(\mathrm{PAN})^{51,52}$ containing BCs. Even though some of these structures might be called worm-like rather than cylindrical, we do not make this distinction here to avoid confusion. More recently, cCCMs could be obtained from BCs with $\pi$-conjugated poly(3-hexylthiophene) (P3HT) as the core-forming block, raising the potential to manufacture conductive and emissive nanowires via direct solution self-assembly as a scalable process (Fig. 1D). ${ }^{\mathbf{4 1 , 5 3}}$ In another approach, the crystallisation of the polyethylene middle block of polystyrene-blockpolyethylene-block-poly(methyl methacrylate) (PS- $b$-PE- $b$ PMMA) triblock terpolymers was used for the preparation of cCCMs. ${ }^{39,54}$ Here, due to the incompatible PS (appears dark) and PMMA (light) segments present within the corona a unique 1D array of alternating patches was observed (Fig. 1B) that could additionally be verified by small-angle neutron scattering. ${ }^{55}$

\section{Pathways of structural control}

For some established systems (PFDMS, PE, PAN) several approaches to tune the structural parameters of the resulting anisotropic particles (length, width, corona composition) have already been developed, and these might also be applicable to similar systems (Scheme 1). Due to the densely packed state of the crystalline core the overall cross-sectional diameter of $1 \mathrm{D}$ CCMs is mainly determined by the length of the corona chains. For a given corona block length, the length of the core block cannot exceed a certain critical value, as this would favour the formation of platelet-like CCMs. ${ }^{25}$ For PFDMS-containing BCs block length ratios of $5 / 1$ (corona/core-forming block) still lead to cCCMs, while for a ratio of $1 / 1$ platelets are formed. ${ }^{33,34}$ In triblock terpolymers with a crystallisable PE middle block, however, block ratios lower than 1/1 still form well-defined 1D structures. ${ }^{39}$ An elegant way to tune the size of the crystalline core was reported by Lazzari and Lopez-Quintela et al., who increased the core diameters of their structures by cocrystallising PAN- $b$-PS with increasing amounts of PAN homopolymer (Scheme 1A). ${ }^{\mathbf{1 1}}$

Another important parameter is the corona composition. While most of the cCCMs produced so far exhibit a homogeneous corona consisting of a single type of polymer chains, routes to surface-compartmentalised elongated micelles have
A) Random Cocrystallisation

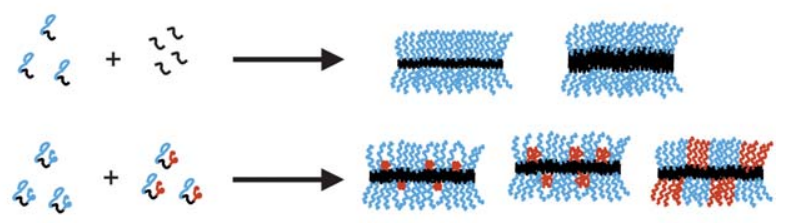

B) Length Control

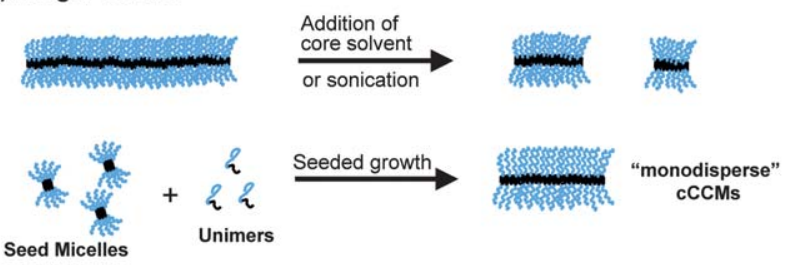

C) Block Cocrystallisation

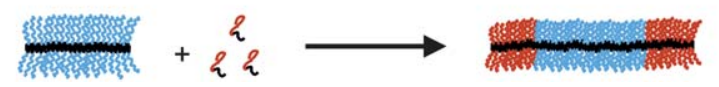

Scheme 1 Control over structural parameters of cCCMs during the self-assembly process: tuning the core width via random cocrystallisation of $A B$ diblock copolymers and homopolymers, or the corona composition by mixing $A B A$ and $A B C$ systems (A); length control via solvent addition, sonication, or seeded growth (B); and generation of block co-micelles via sequential crystallisation (C).

already been explored. In the case of two PFDMS based BCs with amorphous PDMS and PI blocks a random cocrystallisation was reported in hexanes, resulting in cCCMs with tunable corona composition made out of PDMS and PI segments. ${ }^{56}$ However, no microphase separation of the corona could be found in this case. Using a slightly different strategy, corona-compartmentalised cCCMs with tunable PMMA patch sizes could be fabricated by random cocrystallisation of a PS- $b$-PE- $b$-PS triblock copolymer and a PS- $b$-PE- $b$-PMMA triblock terpolymer. The protocol was shown to be facile and robust, allowing for a simple mixing of both components in solution, followed by cooling-induced crystallisation (Scheme 1A). ${ }^{57}$ Block co-micelles with a block-type compartmentalised corona will be addressed in the next section.

Obviously, a very significant property of cCCMs is their length. The use of ultrasound was shown to enable the scission of micelles based on PFDMS and, thus, to tune their length. ${ }^{32,58}$ A similar fragmentation of micelles was observed upon adding trace amounts of a common solvent (Scheme 1B). ${ }^{59}$ For PS- $b$-PE$b$-PMMA cCCMs on the other hand the average micellar length could be tuned by the applied temperature of isothermal crystallisation. ${ }^{39}$ One common drawback of all of these approaches mentioned is that the resulting micelles exhibit a rather high length polydispersity.

This disadvantage was recently overcome by Winnik and Manners et al. by the development of the so-called "living" crystallisation-driven self-assembly. ${ }^{58}$ Here, small stub-like micelles ("crystallites" or seed micelles) are first prepared via sonication of pre-formed cCCMs at low temperatures and afterwards used as seeds for the controlled growth of BC unimers (Scheme 1B). This enabled the production of cCCMs 
with length polydispersities, $L_{\mathrm{w}} / L_{\mathrm{n}}$, down to 1.01 (ref. 60) and number-average lengths, $L_{\mathrm{n}}$, that are determined by the unimer to seed ratio. Meanwhile, living-like self-assembly has also been reported for systems containing crystallisable PFDES, P3HT, srPLA and PE blocks. ${ }^{41,42,46,61}$ Herein, three different methods of seed preparation were applied. Most of the cCCMs were grown from seeds produced via sonication of cylindrical micelles. For PFDMS, a self-seeding approach - the partial dissolution of preformed cylindrical micelles at temperatures slightly below the melting point of PFDMS so that only a few crystalline fragments prevail - has been followed as well. ${ }^{62}$ An alternative way of seed production was developed for PE containing triblock copolymers, which utilizes spherical CCMs pre-assembled in non-solvents for the PE block as seeds. ${ }^{61}$

\section{Complex architectures}

Strikingly similar to well-known living polymerisation techniques, this "living" crystallisation also enables the preparation of block-type co-micelles (Scheme 1C). Upon addition of a second batch of $\mathrm{BC}$ unimers bearing a different corona-forming block these grow epitaxially onto existing cCCMs ("block cocrystallisation") yielding symmetric triblock co-micelles, as shown for PFDMS containing BCs. ${ }^{58}$ By further sequential unimer addition block co-micelles with up to 9 blocks have been produced so far. ${ }^{63}$ Furthermore, BCs containing PFDMG are able to grow heteroepitaxially from cCCMs with a PFDMS core and vice versa (Fig. 2A). ${ }^{45}$ Despite a comparable crystal lattice mismatch to PFDMS as for PFDMG, PFDES BCs could not be grown from PFDMS cCCMs, showing that other factors such as the actual rate of crystallisation may also play an important role. ${ }^{46}$ Even more complex structures could be realized by the epitaxial growth of PFDMS based BC unimers from the corresponding platelet-like CCMs or crystalline homopolymer films on e.g., silicon substrates, resulting in scarf-like micelles (Fig. 2D) and micellar brush layers, respectively. ${ }^{45}$

Another feature that synergistically complements the opportunities given by living self-assembly is the reversible crosslinking of PI coronas using Karstedt's catalyst. ${ }^{64}$ This allows for the selective redissolution of non-crosslinked structural segments while the crosslinked parts remain intact, as shown in the production of scarf-like micelles with a central hole (Fig. 2E). More lately, linearly elongated micelles, branched structures and micellar networks were fabricated from preformed cCCMs (Fig. 2F) by the addition of PFDMS BC unimers with rather low block ratios $(\leq 2 / 1)$ or even PFDMS homopolymer. ${ }^{65}$ Despite the differences in seed preparation, all approaches involving epitaxial growth in solution so far follow bidirectional growth along the long axis of the initial stub, seed, or spherical micelle. Very recently, the first protocol for the preparation of asymmetric $\mathrm{AB}$ and $\mathrm{ABC}$ block co-micelles with a crystalline core was reported and the resulting cCCMs were shown to act as supramolecular amphiphiles, leading to giant supermicelles in selective media (Fig. $2 \mathrm{G}$ and $\mathrm{H}$ ). ${ }^{66}$

The concept of block cocrystallisation was recently extended to triblock copolymers with PE middle blocks, yielding the first purely organic block co-micelles. ${ }^{61}$ PS- $b$-PE- $b$-PS (SES) cCCMs
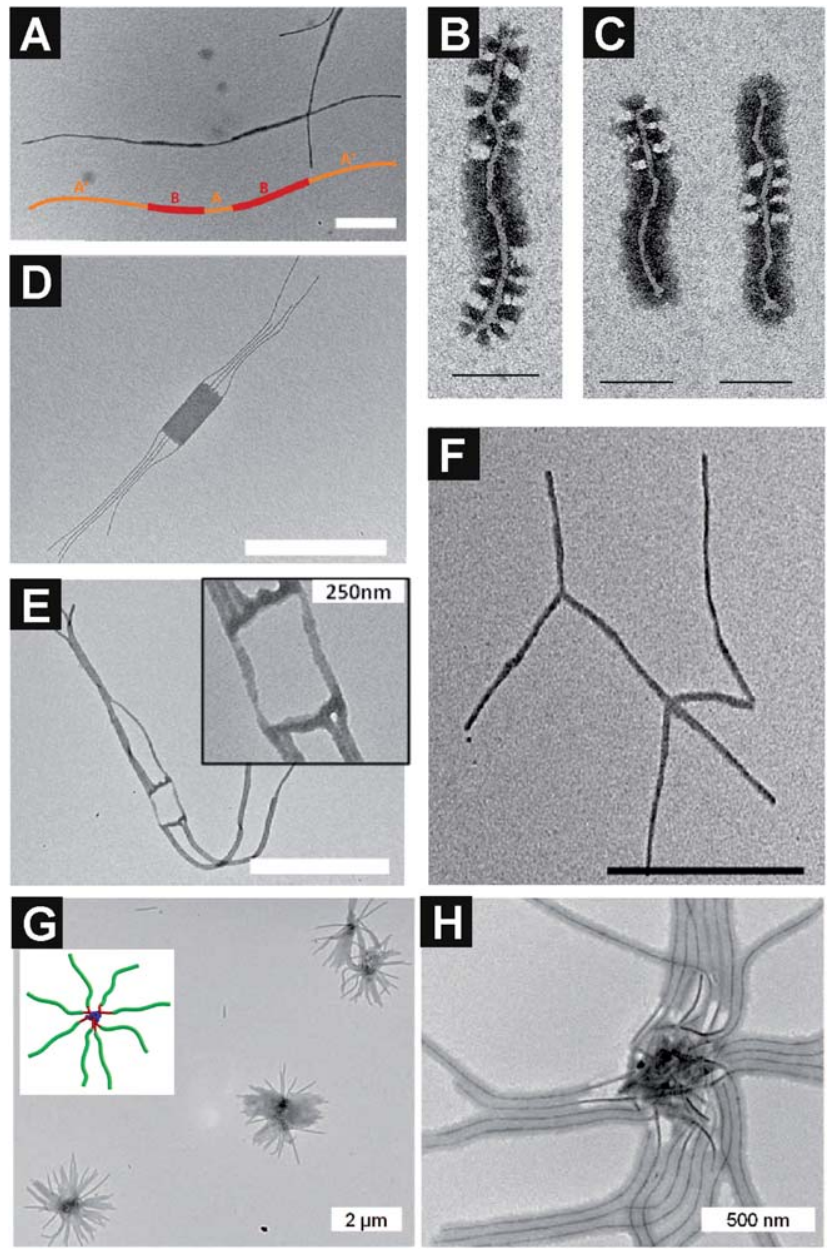

Fig. 2 Block co-micelles obtained via heteroepitaxial growth of PFDMG-containing BCs from micellar seeds with a crystalline PFDMS core (A, adapted from ref. 45 with permission; Copyright 2009 Macmillan Publishers Ltd.); $A B$ and $A B A$ block co-micelles from triblock copolymers with a crystallisable PE middle block ( $B$ and $C$, reprinted with permission from ref. 61; Copyright 2012 American Chemical Society); scarf-like micelles via stepwise self-assembly of PFDMS based BCs (D) and the same structures after crosslinking of the PI corona and selective dissolution of non-crosslinked segments ( $E$, reprinted with permission from ref. 64; Copyright 2011 American Chemical Society); micellar networks from the co-assembly of cCCMs with a PFDMS core and PFDMS homopolymer ( $F$, reprinted with permission from ref. 65; Copyright 2011 American Chemical Society); supermicelles generated via the hierarchical self-assembly of asymmetric ABC triblock co-micelles ( $G, H$, adapted from ref. 66 with permission; Copyright 2012 American Association for the Advancement of Science).

were used as seeds for the epitaxial growth of a PS- $b$-PE- $b$-PMMA (SEM) triblock terpolymer to produce SEM- $b$-SES- $b$-SEM (ABAtype) triblock co-micelles with a complex corona structure almost quantitatively (Fig. 2B). Here, middle blocks with a homogeneous PS corona are surrounded by two outer blocks bearing a patch-like corona that consists of alternating PS and PMMA compartments. In contrast, in the reversed case (epitaxial growth of SES onto SEM CCCM seeds) the blocking efficiency is significantly lower, resulting in a mixture of triblock and diblock co-micelles (Fig. 2C) together with remaining SEM cCCMs. This asymmetric behaviour was attributed to the different corona structure of the applied seeds, however, the exact mechanism still remains a matter of debate. 


\section{Applications}

Despite that this research field is relatively new (10-15 years), the potential of cCCMs has already been explored in view of several applications. One intriguing aspect is that the processes involved are rather simple and, furthermore, unprecedented geometries can be accessed. Such novel structures represent ideal templates for the production of functional hybrid materials or the directed incorporation of metal nanoparticles, dyes, and/or drugs in confined areas, given that corona blocks carrying the required functional groups are present. For $\mathrm{Au}$ and $\mathrm{PbS}$ nanoparticles the selective deposition in PFDMS containing ABA block co-micelles with a central quaternised P2VP corona block (Pq2VP) was accomplished, while the non-quaternised P2VP outer blocks remained unaffected (Fig. 3A). ${ }^{67}$

Using a similar approach, continuous and segmented metal oxide/polymer nanowires with surfaces consisting of e.g., zirconia, alumina or titania (Fig. 3B) were manufactured via a template-directed sol-gel process. ${ }^{68}$ Furthermore, 9block co-micelles with fluorescent monomers in the corona of every second corona block were proposed as micellar "barcodes" (Fig. 3C). ${ }^{63}$ In order to fabricate carbon nanofibers, sacrificial PAN-based cCCMs with tunable cores were subjected to pyrolysis (Fig. 3D). ${ }^{51}$ The resulting carbon nanofibers finally exhibit half of the diameter of the original micellar core.
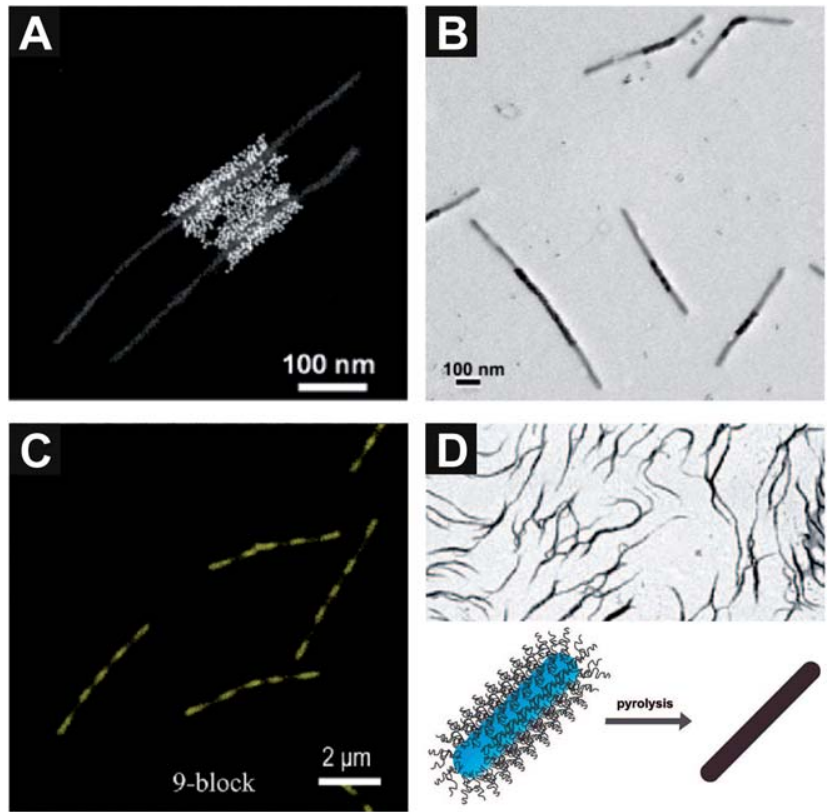

Fig. 3 ABA block co-micelles with a central quaternised Pq2VP corona segment after selective loading with Au nanoparticles (A, reprinted with permission from ref. 67; Copyright 2007 American Chemical Society); segmented polymer/metal oxide nanowires with a titania surface ( $B$, adapted from ref. 68 with permission); fluorescent 9-block co-micelles as micellar "barcodes" (C, reprinted with permission from ref. 63; Copyright 2011 American Chemical Society); PAN-based cCCMs, which can be transformed into carbon nanofibers by pyrolysis ( $D$, reprinted with permission from ref. 51; Copyright 2007 American Chemical Society).

\section{Conclusions and future perspective}

Crystallisation-induced self-assembly of BCs in solution is a powerful tool for the production of cCCMs with well-defined lengths and length distributions as well as corona compositions, owing to the living nature of the process. The observation that the concept of "living" crystallisation-driven self-assembly can be applied to $\mathrm{PE}$ containing $\mathrm{BCs},{ }^{61}$ i.e., $\mathrm{BCs}$ bearing a crystallisable block with inherent structural imperfection (ethyl branches) that most of the common crystallisable polymers do not share, shows that this concept should be generally applicable to semicrystalline BCs. On the other hand, it remains a challenge to achieve perfect heteroepitaxial growth into core-compartmentalised cCCMs. So far, this process could only be realized for combinations of PFDMS and PFDMG,${ }^{45}$ whereas only subtle changes, i.e., the use of longer alkyl chains in PFDES, resulted in unsuccessful cocrystallisation attempts. ${ }^{46}$ Clearly, lattice mismatch is not the only criterion as the differences in lattice parameter between PFDMS/PFDES and PFDMS/PFDMG according to X-ray studies were comparable. Presumably, crystallisation kinetics and steric hindrance during chain folding play important roles as well. One strategy may be to target epitaxial compatibilizers, thereby generating a gradient crossover between unlike crystalline core-forming segments.

Nevertheless, crystallisation gave access to more complex architectures, e.g., symmetric and asymmetric block comicelles, block co-micelles with homogeneous and/or patchy corona blocks and scarf-like micelles. We strongly believe that such systems represent attractive model systems or templates for a wide range of applications in the near future.

For surface compartmentalised block-type architectures or inherently patch-like cCCMs from triblock terpolymers an increased surface activity as well as a strong tendency to further assemble into ordered superstructures can be expected. In analogy to liquid-crystalline rod-like molecules, cCCMs with low length polydispersities might also be able to form ordered phases in more concentrated solutions with potential in flowdependent applications. ${ }^{69}$ Well-defined cylinders of comparably low aspect ratio could be highly interesting as drug carriers due to their prolonged blood circulation time. ${ }^{70}$ However, finding suitable systems that combine all of the necessary features, i.e., biocompatibility, structural stability in the cellular environment and coronas suitable for drug uptake and release, will be one of the main future challenges.

\section{Acknowledgements}

JS and HS appreciate support by the German Science Foundation in the framework of the Collaborative Research Centre SFB 840 (project A2). FHS is grateful to the FCI for a fellowship and also to the Thuringian Ministry for Education, Science and Culture (TMBWK, grant \#B514-09051, NanoConSens) for financial support.

\section{Notes and references}

1 M. Lazzari and M. A. Lopez-Quintela, Adv. Mater., 2003, 15, 1583-1594. 
2 J. Rodríguez-Hernández, F. Chécot, Y. Gnanou and S. Lecommandoux, Prog. Polym. Sci., 2005, 30, 691-724.

3 G. Riess, Prog. Polym. Sci., 2003, 28, 1107-1170.

4 B. M. Discher, Y.-Y. Won, D. S. Ege, J. C.-M. Lee, F. S. Bates, D. E. Discher and D. A. Hammer, Science, 1999, 284, 11431146.

5 L. Zhang and A. Eisenberg, Science, 1995, 268, 1728-1731.

6 F. H. Schacher, P. A. Rupar and I. Manners, Angew. Chem., Int. Ed., 2012, 51, 7898-7921.

7 A. Walther, M. Hoffmann and A. H. E. Müller, Angew. Chem., Int. Ed., 2008, 47, 711-714.

8 M. Hamidi, M. A. Shahbazi and K. Rostamizadeh, Macromol. Biosci., 2012, 12, 144-164.

9 A. V. Ruzette and L. Leibler, Nat. Mater., 2005, 4, 19-31.

10 A. Walther, K. Matussek and A. H. E. Müller, ACS Nano, 2008, 2, 1167-1178.

11 H. Ruckdäschel, J. K. W. Sandler, V. Altstädt, H. Schmalz, V. Abetz and A. H. E. Müller, Polymer, 2007, 48, 27002719.

12 E. A. Jackson and M. A. Hillmyer, ACS Nano, 2010, 4, 35483553.

13 C. H. Braun, T. V. Richter, F. Schacher, A. H. E. Müller, E. J. W. Crossland and S. Ludwigs, Macromol. Rapid Commun., 2010, 31, 729-734.

14 Y. Liu, Y. Wang, Y. F. Wang, J. Lu, V. Pinon and M. Weck, J. Am. Chem. Soc., 2011, 133, 14260-14263.

15 R. C. Hayward and D. J. Pochan, Macromolecules, 2010, 43, 3577-3584.

16 A. O. Moughton, M. A. Hillmyer and T. P. Lodge, Macromolecules, 2012, 45, 2-19.

17 E. B. Zhulina and O. V. Borisov, Macromolecules, 2012, 45, 4429-4440.

18 P. Bhargava, Y. Tu, J. X. Zheng, H. Xiong, R. P. Quirk and S. Z. D. Cheng, J. Am. Chem. Soc., 2007, 129, 1113-1121.

19 W. Zhang, M. Wang and K. L. Wooley, Curr. Org. Chem., 2005, 9, 1053-1066.

20 A. H. Gröschel, F. H. Schacher, H. Schmalz, O. V. Borisov, E. B. Zhulina, A. Walther and A. H. E. Müller, Nat. Commun., 2012, 3, 710.

21 H. Cui, Z. Chen, S. Zhong, K. L. Wooley and D. J. Pochan, Science, 2007, 317, 647-650.

22 A. Wolf, A. Walther and A. H. E. Müller, Macromolecules, 2011, 44, 9221-9229.

23 Y. Liu, V. Abetz and A. H. E. Müller, Macromolecules, 2003, 36, 7894-7898.

24 F. H. Schacher, T. Rudolph, M. Drechsler and A. H. E. Müller, Nanoscale, 2011, 3, 288-297.

25 T. Vilgis and A. Halperin, Macromolecules, 1991, 24, 20902095.

26 E. K. Lin and A. P. Gast, Macromolecules, 1996, 29, 44324441.

27 M. Dröscher and T. L. Smith, Macromolecules, 1982, 15, 442449.

28 K. A. Cogan and A. P. Gast, Macromolecules, 1990, 23, 745753.

29 A. P. Gast, P. K. Vinson and K. A. Cogan-Farinas, Macromolecules, 1993, 26, 1774-1776.
30 D. Richter, D. Schneiders, M. Monkenbusch, L. Willner, L. J. Fetters, J. S. Huang, M. Lin, K. Mortensen and B. Farago, Macromolecules, 1997, 30, 1053-1068.

31 W.-N. He and J.-T. Xu, Prog. Polym. Sci., 2012, 37, 13501400.

32 J. Massey, K. N. Power, I. Manners and M. A. Winnik, J. Am. Chem. Soc., 1998, 120, 9533-9540.

33 J. A. Massey, K. Temple, L. Cao, Y. Rharbi, J. Raez, M. A. Winnik and I. Manners, J. Am. Chem. Soc., 2000, 122, 11577-11584.

34 L. Cao, I. Manners and M. A. Winnik, Macromolecules, 2002, 35, 8258-8260.

35 I. Korczagin, M. A. Hempenius, R. G. Fokkink, M. A. Cohen Stuart, M. Al-Hussein, P. H. H. Bomans, P. M. Frederik and G. J. Vancso, Macromolecules, 2006, 39, 2306-2315.

36 H. Wang, M. A. Winnik and I. Manners, Macromolecules, 2007, 40, 3784-3789.

37 J.-F. Gohy, B. G. G. Lohmeijer, A. Alexeev, X.-S. Wang, I. Manners, M. A. Winnik and U. S. Schubert, Chem.-Eur. J., 2004, 10, 4315-4323.

38 X. Wang, M. A. Winnik and I. Manners, Macromolecules, 2005, 38, 1928-1935.

39 J. Schmelz, M. Karg, T. Hellweg and H. Schmalz, ACS Nano, 2011, 5, 9523-9534.

40 W. N. He, J. T. Xu, B. Y. Du, Z. Q. Fan and X. S. Wang, Macromol. Chem. Phys., 2010, 211, 1909-1916.

41 S. K. Patra, R. Ahmed, G. R. Whittell, D. J. Lunn, E. L. Dunphy, M. A. Winnik and I. Manners, J. Am. Chem. Soc., 2011, 133, 8842-8845.

42 N. Petzetakis, A. P. Dove and R. K. O'Reilly, Chem. Sci., 2011, 2, 955-960.

43 A. M. Mihut, J. J. Crassous, H. Schmalz, M. Drechsler and M. Ballauff, Soft Matter, 2012, 8, 3163-3173.

44 M. Lazzari and M. A. Lopez-Quintela, Macromol. Rapid Commun., 2009, 30, 1785-1791.

45 T. Gädt, N. S. Ieong, G. Cambridge, M. A. Winnik and I. Manners, Nat. Mater., 2009, 8, 144-150.

46 T. Gädt, F. H. Schacher, N. McGrath, M. A. Winnik and I. Manners, Macromolecules, 2011, 44, 3777-3786.

47 Z.-X. Du, J.-T. Xu and Z.-Q. Fan, Macromolecules, 2007, 40, 7633-7637.

48 D. Portinha, F. Boué, L. Bouteiller, G. Carrot, C. Chassenieux, S. Pensec and G. Reiter, Macromolecules, 2007, 40, 40374042.

49 N. Petzetakis, D. Walker, A. P. Dove and R. K. O'Reilly, Soft Matter, 2012, 8, 7408-7414.

50 A. M. Mihut, M. Drechsler, M. Möller and M. Ballauff, Macromol. Rapid Commun., 2010, 31, 449-453.

51 M. Lazzari, D. Scalarone, C. E. Hoppe, C. Vazquez-Vazquez and M. A. Lopez-Quintela, Chem. Mater., 2007, 19, 58185820.

52 M. Lazzari, D. Scalarone, C. Vazquez-Vazquez and M. A. Lopez-Quintela, Macromol. Rapid Commun., 2008, 29, 352-357.

53 J. B. Gilroy, D. J. Lunn, S. K. Patra, G. R. Whittell, M. A. Winnik and I. Manners, Macromolecules, 2012, 45, 5806-5815. 
54 H. Schmalz, J. Schmelz, M. Drechsler, J. Yuan, A. Walther, K. Schweimer and A. M. Mihut, Macromolecules, 2008, 41, 3235-3242.

55 S. Rosenfeldt, F. Lüdel, C. Schulreich, T. Hellweg, A. Radulescu, J. Schmelz, H. Schmalz and L. Harnau, Phys. Chem. Chem. Phys., 2012, 14, 12750-12756.

56 G. Cambridge, G. Guerin, I. Manners and M. A. Winnik, Macromol. Rapid Commun., 2010, 31, 934-938.

57 J. Schmelz and H. Schmalz, Polymer, 2012, 53, 4333-4337.

58 X. Wang, G. Guerin, H. Wang, Y. Wang, I. Manners and M. A. Winnik, Science, 2007, 317, 644-647.

59 J. Qian, G. Guerin, G. Cambridge, I. Manners and M. A. Winnik, Macromol. Rapid Commun., 2010, 31, 928933.

60 J. B. Gilroy, T. Gädt, G. R. Whittell, L. Chabanne, J. M. Mitchels, R. M. Richardson, M. A. Winnik and I. Manners, Nat. Chem., 2010, 2, 566-570.

61 J. Schmelz, A. E. Schedl, C. Steinlein, I. Manners and H. Schmalz, J. Am. Chem. Soc., 2012, 134, 14217-14225.
62 J. Qian, G. Guerin, Y. Lu, G. Cambridge, I. Manners and M. A. Winnik, Angew. Chem., Int. Ed., 2011, 50, 1622-1625.

63 F. He, T. Gädt, I. Manners and M. A. Winnik, J. Am. Chem. Soc., 2011, 133, 9095-9103.

64 P. A. Rupar, G. Cambridge, M. A. Winnik and I. Manners, J. Am. Chem. Soc., 2011, 133, 16947-16957.

65 S. F. Mohd Yusoff, J. B. Gilroy, G. Cambridge, M. A. Winnik and I. Manners, J. Am. Chem. Soc., 2011, 133, 11220-11230.

66 P. A. Rupar, L. Chabanne, M. A. Winnik and I. Manners, Science, 2012, 337, 559-562.

67 H. Wang, W. Lin, K. P. Fritz, G. D. Scholes, M. A. Winnik and I. Manners, J. Am. Chem. Soc., 2007, 129, 12924-12925.

68 H. Wang, A. J. Patil, K. Liu, S. Petrov, S. Mann, M. A. Winnik and I. Manners, Adv. Mater., 2009, 21, 1805-1808.

69 J. B. Gilroy, P. A. Rupar, G. R. Whittell, L. Chabanne, N. J. Terrill, M. A. Winnik, I. Manners and R. M. Richardson, J. Am. Chem. Soc., 2011, 133, 17056-17062.

70 Y. Geng, P. Dalhaimer, S. Cai, R. Tsai, M. Tewari, T. Minko and D. E. Discher, Nat. Nanotechnol., 2007, 2, 249-255. 